$$
\text { CONF-960569--10 }
$$

\title{
Simulation of the Scrape-Off Layer Plasma During a Disruption
}

\author{
T.D. Rognlien \\ J.A. Crotinger \\ G.D. Porter \\ G.R. Smith \\ A.G. Kellman \\ P.L. Taylor
}

\author{
PECERED \\ SEP 09 1986 \\ OSTI
}

This paper was prepared for submittal to

12th International Conference on Plasma Surface Interactions in Controlled Fusion Devices

Saint-Raphael, France

May 20-24, 1996

March 16, 1996

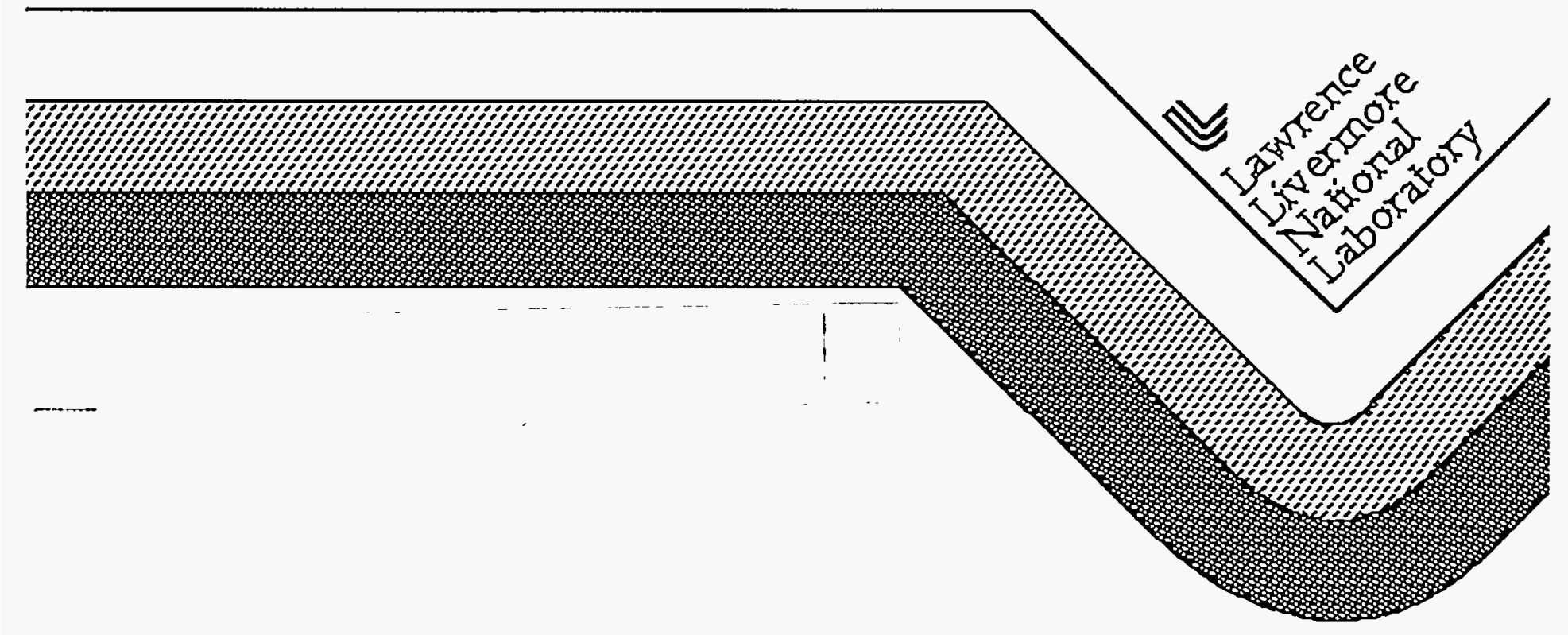




\section{DISCLAIMER}

This document was prepared as an account of work sponsored by an agency of the United States Government. Neither the United States Government nor the University of California nor any of their employees, makes any warranty, express or implied, or assumes any legal liability or responsibility for the accuracy, completeness, or usefulness of any information, apparatus, product, or process disclosed, or represents that its use would not infringe privately owned rights. Reference herein to any specific commercial product, process, or service by trade name, trademark, manufacturer, or otherwise, does not necessarily constitute or imply its endorsement, recommendation, or favoring by the United States Government or the University of California. The views and opinions of authors expressed herein do not necessarily state or reflect those of the United States Government or the University of California, and shall not be used for advertising or product endorsement purposes. 


\section{DISCLAIMER}

Portions of this document may be illegible in electronic image products. Images are produced from the best available original document. 


\title{
Simulation of the Scrape-Off Layer Plasma During a Disruption
}

\author{
T.D. Rognlien, J.A. Crotinger, G.D. Porter, and G.R. Smith \\ Lawrence Livermore National Laboratory, \\ Livermore, California 94551 USA.
}

\author{
A.G. Kellman and P.L. Taylor \\ General Atomics, San Diego, CA 92186 USA
}

The evolution of the scrape-off layer (SOL) during a disruption in the DIII-D tokamak is modeled using the 2-D UEDGE transport code. The focus is on the thermal quench phase when most of the energy content of the discharge is rapidly transported across the magnetic separatrix where it then flows to material surfaces or is radiated. Comparisons between the simulation and an experiment on the DIII-D tokamak are made with the heat flux to the divertor plate, and temperature and density profiles at the SOL midplane. The temporal response of the separate electron and ion heat-flux components to the divertor plate is calculated. The sensitivity of the solution to assumptions of electron heat-flux models and impurity radiation is investigated. 


\section{Introduction}

Disruptions in tokamaks can often be characterized by two periods: the first is the thermal quench where the plasma energy of the core is lost, and the second is the current-decay where the plasma current is lost [1]. The scrape-off layer (SOL) plasma takes on an important role during the thermal quench of a tokamak disruption. Specifically, the SOL determines how the power from the core plasma is distributed on material surfaces, how impurities radiation affects the power loss, and the properties of the SOL for carrying halo currents. The location and temporal variation of the heat load on surfaces are important input for material ablation calculations [2]. In addition to the large heat fluxes, the plasma current is typically shunted through the vacuum vessel in the current-decay phase, causing large $\mathbf{J} \times \mathbf{B}$ forces on the vessel. Also, the large inductive electric field during the current-decay phase can produce damaging run-away electrons. All of these consequences are of concern for large future devices such as ITER.

In an effort to better understand the energy loss during the thermal quench, we simulate the SOL plasma for the DIII-D tokamak $[3,4]$ in a time-dependent fashion using the SOL transport code UEDGE [5,6]. Our primary focus here is on the initial thermal quench phase when most of the core energy is lost, but the total current and the shape of the MHD equilibrium is maintained. In Sec. 2, we describe the experimentally measured characteristics of a disruption in DIII-D, and the simulation model is given in Sec. 3. Section 4 contains a comparison between the experiment and the simulation where we discussion the importance of assumptions in the model, and we give a summary in Sec. 4.

\section{Experimental measurements during a disruption}

Various types of disruptions can occur in the DIII-D tokamak, e.g., from puffing deuterium or impurity gas and from exceeding a plasma $\beta$-limit. Impurity induced disruptions in DIII-D are described elsewhere $[3,4]$. Here we consider a $\beta$-limit disruption that occurred on discharge 84539. This single-null divertor discharge had a plasma current of 1.5 MA, a toroidal magnetic field of $1.2 \mathrm{~T}$, and a $q$ safety factor of $\sim 2.5$. Neutral beam injection was ramped up to $17 \mathrm{MW}$ before the disruption near $2.2 \mathrm{~s}$ into the discharge. In Fig. 1, we show the plasma current $\left(I_{p}\right)$ and the central soft x-ray signal (measuring the central electron temperature, $T_{e c}$ ) near the time of the disruption. First, the plasma energy in the core decays (measured by $T_{e c}$ ), followed by the decay of $I_{p}$.

During the time of the thermal quench ( $2.20 \mathrm{~s}$ to $2.21 \mathrm{~s})$, the heat flux on the divertor plates rises rapidly and broadens as shown in Fig. 2. The inner and outer 
plate separatrix strike points are denoted by the dashed lines on the major axis coordinate, $R$. Note that the heat flux is predominately on the SOL sides of the strike points. Also, often the temporal behavior is characterized by bursts with widths of 0.5-1 ms, which is about the temporal resolution of the instrument.

The electron temperature $\left(T_{e}\right)$ and density $(n)$ are measured by a Thomson scattering system near the outer midplane. Three time-slices are shown in Fig. 3, the first being before the disruption, the second is half-way through the thermal quench and the last is at the end of the thermal quench. Note the broadening of the SOL and the decrease in the temperature deeper into the core. Because of the scale, the first two time-slices do not show the maximum $T_{e}$ measured (near $r / a=0.4$ ): at $t=2.1860 \mathrm{~s}$, $T_{e-\max } \approx 1.5 \mathrm{keV}$ and at $t=2.2055 \mathrm{~s}, T_{e-\max } \approx 1.0 \mathrm{keV}$. There are charge-exchange recombination (CER) measurements of the ion temperature, $T_{i}$, which is typically a factor of two or more above $T_{e}$.

\section{Numerical model}

The UEDGE code solves for the plasma fluid equations in the 2-D poloidal plane, including the region somewhat inside the separatrix and extending into the SOL. A finite volume discretization method is used, and the resulting difference equations are solved with a fully implicit Newton method $[5,6]$. The code has been used in a steady-state mode to model many normal (non-disrupting) DIII-D discharges [9]. The 2-D mesh is derived from the poloidal magnetic flux surfaces as one coordinate which are constructed by the GA EFIT code. With the poloidal flux, $\psi$, normalized to unity on the separatrix, we take the inner simulation boundary to be $\psi_{c}=0.98$ and the outer boundary at $\psi_{w}=1.10$; the private flux region is also included.

Plasma equations are solved for the ion density, $n_{i}$, and ion parallel velocity $v_{i}$, and separate equations are used for the electron and ion temperatures, $T_{e}$ and $T_{i}$. Poloidal transport is the geometrical projection of the parallel transport from Braginskii [7], except that thermal flux limits are used as discussed below. Radial transport is described by anomalous diffusion coefficients chosen to fit experimental data. We can use separate diffusion coefficients for density $(D)$, electron energy $\left(\chi_{e}\right)$, ion energy $\left(\chi_{i}\right)$. The neutral species is modeled by a simple 2-D diffusive fluid equation for the present calculations. Atomic physics rate coefficients for ionization, recombination, and the associated energy loss are provided by look-up tables as described elsewhere [8].

The practice of adjusting the radial diffusion coefficients to obtain a fit to experimental data requires some comment for the case of disruptions where the details of the transport are not well understood and may not even be diffusive. However, given 
that the plasma energy is somehow transported to open magnetic field lines in the thermal quench time, we can study the 2-D effect of the competition between the radial flux and the assumed classical parallel transport to see if a consistent picture evolves.

One correction to the classical parallel transport that we consider is limiting the thermal heat flux. With the diffusive thermal fluxes being $q_{e, i}=-\kappa_{e, i} \partial T_{e, i} / \partial s$, the modification is as follows

$$
\kappa_{e, i} \rightarrow \frac{\kappa_{e, i}}{\left(1+\left|q_{e, i}\right| / q_{f}\right)} .
$$

Here $q_{f}=c_{f} n v_{t e, t i} T_{e, i}$ is the thermal flux reduced by the coefficient $c_{f}$. More discussion of flux limiting for normal discharge parameters is given elsewhere [10]. However, for disruptions, little is know of the particle distribution functions. Therefore, we shall treat $c_{f}$ unknown, and investigate the effect of taking different values.

\section{Simulation of the SOL and comparison with the experi- ment}

We obtain a pre-disruption equilibrium with UEDGE by using the anomalous diffusion coefficients of $D=\chi_{e}=\chi_{i}=1 \mathrm{~m}^{2} / \mathrm{s}$. While these give fair comparison with the data, no attempt was made to fit the pre-disruption data precisely as we focus on the disruption period. The recycling coefficient at the plates are set at 0.95 throughout the simulations. As can be seen from Fig. 1, the energy of the plasma is lost in a time of about $10 \mathrm{~ms}$ and the energy striking the plates in this time is $\sim 1 \mathrm{MJ}$. Allowing for a $50 \%$ duty factor for the load on the plates (see Fig. 2, we translate this into a boundary condition at the core edge for UEDGE of $1 \mathrm{MJ} /(0.5 \times 10 \mathrm{~ms})=$ $200 \mathrm{MW}$, which we divide equally between the ions and electrons. With this boundary condition, UEDGE evolves the SOL plasma to equilibrium.

We have run a number of simulations with different anomalous diffusion coefficients to fit the shape of the divertor heat-flux profiles shown in Fig. 2 and find that a reasonable fit is found for $D=\chi_{e}=\chi_{i}=20 \mathrm{~m}^{2} / \mathrm{s}$. The temporal evolution of the heat flux profiles on the inner and outer divertor plates is shown in Fig. 4. The five rising curves correspond in order to increasing times from the initiation of the disruption (see caption). Thus, the increased heat flux first arrives at the outer plate, it being closer to the outer midplane where a majority of the power is injected into the SOL owing to the more compressed flux surfaces there. The flux on both plates is close to steady state by $t=0.3 \mathrm{~ms}$, and the total power to each plate is nearly the same. The time scale and shape are consistent with the experimental profiles shown in Fig. 2. There is even a suggestion of the heat pulses being narrower and more peaked on the inner plate, but the data is too varied for a clear conclusion. 
The inner/outer asymmetries may also have to do with toroidal variations that we do not consider. We did include parallel electric currents from electrostatic fields which lead to small changes in the asymmetry for the disruption period, but do enhance the outer plate power by $30 \%$ for the pre-disruption equilibrium. We plan to extend this to inductive fields.

The total heat-flux profiles are not too sensitive to the choice of the flux-limit factors, $c_{f}$, for the electrons and ions; the results in Fig. 4 has $c_{f e}=0.1$ for electrons and $c_{f i} \rightarrow \infty$ for ions. The plate temperatures do decrease with $c_{f}$ somewhat, but the midplane values where we have the Thomson data are not strongly affected. The peak plate $T_{e}$ is about $250 \mathrm{eV}$ without flux-limiting and $200 \mathrm{eV}$ with $c_{f e}=0.1$. An enhanced energy transmission factor from kinetic effects decreases the plate temperatures; here we have used standard values of 4.0 for electrons and 2.5 for ions. Another effect reducing $T_{e}$ at the plate is impurity radiation.

Bolometer measurements indicate that 30-40\% of the energy is radiated during the disruption. We have modeled this by using a fixed-fraction model of carbon impurities corresponding to a well-mixed plasma. In order to get $\sim 30 \%$ of the power radiated, we need a carbon fraction of $25 \%$. The radiation occurs close to the divertor plates, but peaks about $6 \mathrm{~cm}$ away from the separatrix where the plate $T_{e}$ is reduced to $\sim 10 \mathrm{eV}$; in this case, the peak plate $T_{e}$ is reduced to $100 \mathrm{eV}$. The effect on the plasma heat-flux profile is shown in Fig. 5; the absorption of the impurity radiation on the plate is not included, but would broaden the profile modestly. In the current decay phase, it will be important to evolve the impurity species [1].

Direct comparison between midplane experimental data of $T_{e}$ and $T_{i}$ and the simulation with $25 \%$ carbon is shown in Fig. 6. The dotted line for $T_{i}$ for $\delta R=$ $R-R_{\text {sep }}<0$ does connect to a data point not shown. The comparison would fit better if the separatrix position were moved about $1 \mathrm{~cm}$, shifting the experimental data outward. Such a shift is not unreasonable given the uncertainty in the data. The high experimental $T_{i}$ at $\delta R=R-R_{\text {sep }}=2.6 \mathrm{~cm}$ appears similar on many timeslices, and may be erroneous. The experimental density (not shown) decays a factor of two over the simulation region while the simulation density only decays about $20 \%$. The effect of removing the impurity radiation is to increase the midplane simulation temperatures by $30-40 \%$ in the SOL region.

\section{Summary}

We have simulated the thermal quench phase of a high- $\beta$ disruption in DIII$D$ using the UEDGE transport code. Choosing anomalous diffusion coefficients of $20 \mathrm{~m}^{2} / \mathrm{s}$ gives a reasonable fit to the divertor heat-flux profiles. The response time 
of $\sim 0.3 \mathrm{~ms}$ is roughly consistent with the data. Kinetic effects such as thermal flux limits and enhance energy transmission primarily affect the temperatures on the plates, and not the heat flux profiles. Impurity radiation also reduces the plate $T_{e}$ and narrows the heat flux profile. Rough agreement is found for the midplane temperature profiles and shifting the separatrix $1 \mathrm{~cm}$ would improve this.

These calculations show the feasibility of modeling a number of the SOL aspects of disruptions with a transport code that could be used in ablation studies; the detailed nature of the escaping energy spectrum requires a kinetic treatment. Important additional work is to assess the response of the growing SOL to carrying current induced by the rising loop voltage.

\section{Acknowledgments}

We gratefully acknowledge discussions with P.N. Brown, R.H. Cohen, A.C. Hindmarsh, L.D. Pearlstein, M.E. Rensink, and A. Tarditi.

This work was performed under the auspices of the U.S. Department of Energy by Lawrence Livermore National Laboratory under contract No. W-7405-Eng-48, and at General Atomics under contract No. DE-AC03-89ER51114. 


\section{References}

[1] D.J. Ward and J.A. Wesson, Nucl. Fusion 32 (1992) 1117.

[2] For example, see A.E. Koniges, D.C. Eder, H.A. Scott, H. Würz, and F. Kappler, this PSI conference, 1996.

[3] P.L. Taylor, A.G. Kellman, B.W. Rice, and D.A Humphreys, General Atomics Rept. GA-A22076, Sept. 1996.

[4] P.L. Taylor, A.G. Kellman, B.W. Rice, et al., $22^{\text {th }}$ EPS Conf. Contr.Fusion and Plasma Phys., Bournemouth, UK, 1995 (also, Rept. GA-A22082).

[5] T.D. Rognlien, P.N. Brown, R.B. Campbell, et al., Contr. Plasma Phys. 34 (1994) 362.

[6] G.R. Smith, P.N. Brown, R.B. Campbell, D.A. Knoll, P.R. McHugh, M.E. Rensink, and T.D. Rognlien, J. Nucl. Mat. 220-222 (1995) 1024.

[7] S.I. Braginskii, Transport processes in a plasma, Reviews of Plasma Physics, Vol. 1, Ed. M.A. Leontovich (Consultants Bureau, New York, 1965), p. 205.

[8] T.D. Rognlien, B.J. Braams, and D.A. Knoll, Contrib. Plasma Phys. 36, to be pub., 1996.

[9] G.D. Porter, et al., Phys. Plasmas 3, to be pub., 1996.

[10] R.H. Cohen and T.D. Rognlien, Contrib. Plasma Phys. 34, (1994) 198. 


\section{Figures}

Fig. 1. Variation of plasma current and central soft x-ray signal measuring $T_{e c}$ for DIII-D discharge 84359.

Fig. 2. Temporal and spatial variation of divertor plate heat flux as measured by the IRTV system for discharge 84359 . The dashed lines denote the approximate location of the inner and outer separatrix strike points.

Fig. 3. Electron temperature $(T)$ and density $(n)$ near the edge region of the midplane as measured by Thomson scattering at various times.

Fig. 4. Time history of heat fiux on the inner and outer divertor plates from UEDGE with $D=\chi_{e}=\chi_{i}=20 \mathrm{~m}^{2} / \mathrm{s}$. The five curves with increasing heat flux correspond respectively to the times $0 \mathrm{~s}, 0.03 \mathrm{~ms}, 0.1 \mathrm{~ms}, 0.3 \mathrm{~ms}$, and steady-state.

Fig. 5. Plasma heat flux on outer plate with and without $25 \%$ fixed-fraction of carbon (coronal model).

Fig. 6. Comparison of $T_{e}$ (Thomson scattering) and $T_{i}$ (CER) at the midplane for $t=2205 \mathrm{~s}$ (lines with data points) with the UEDGE simulation (lines without data points). 


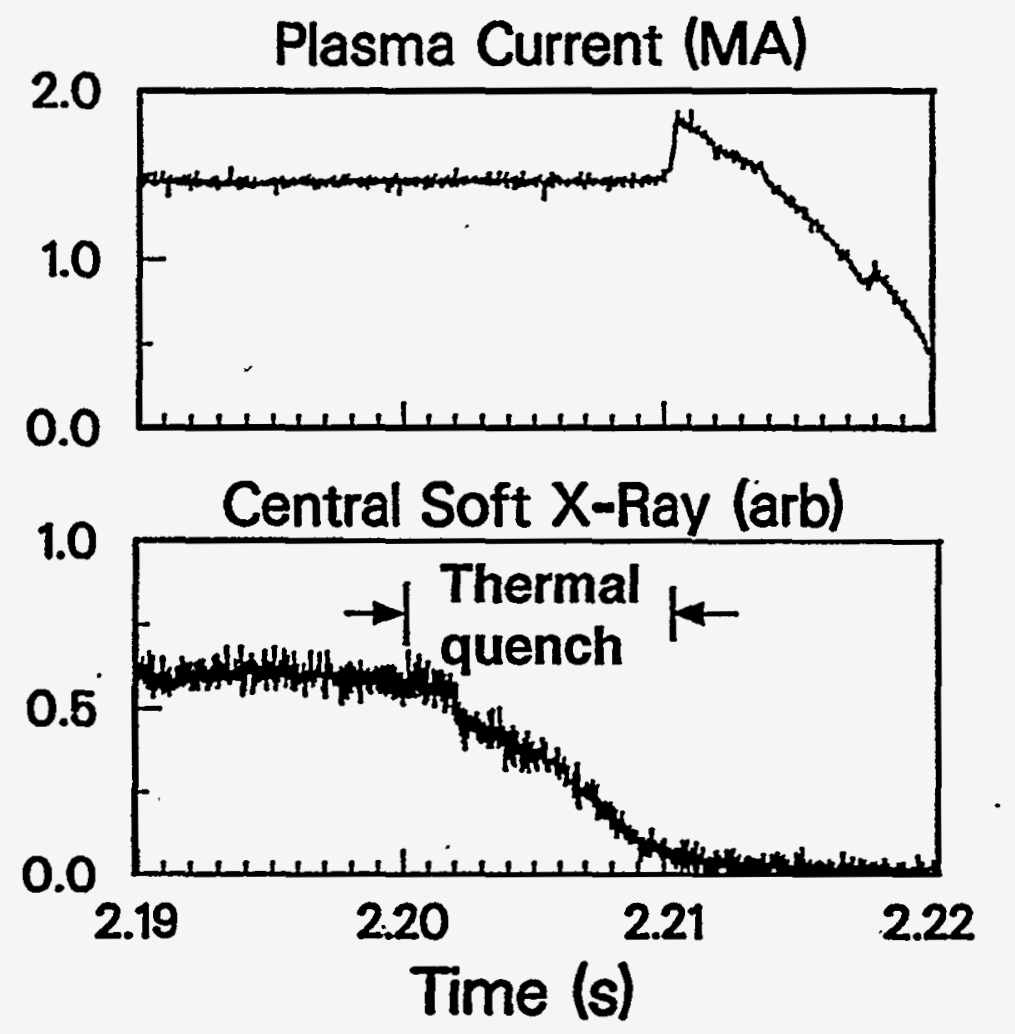

Fig. 1 


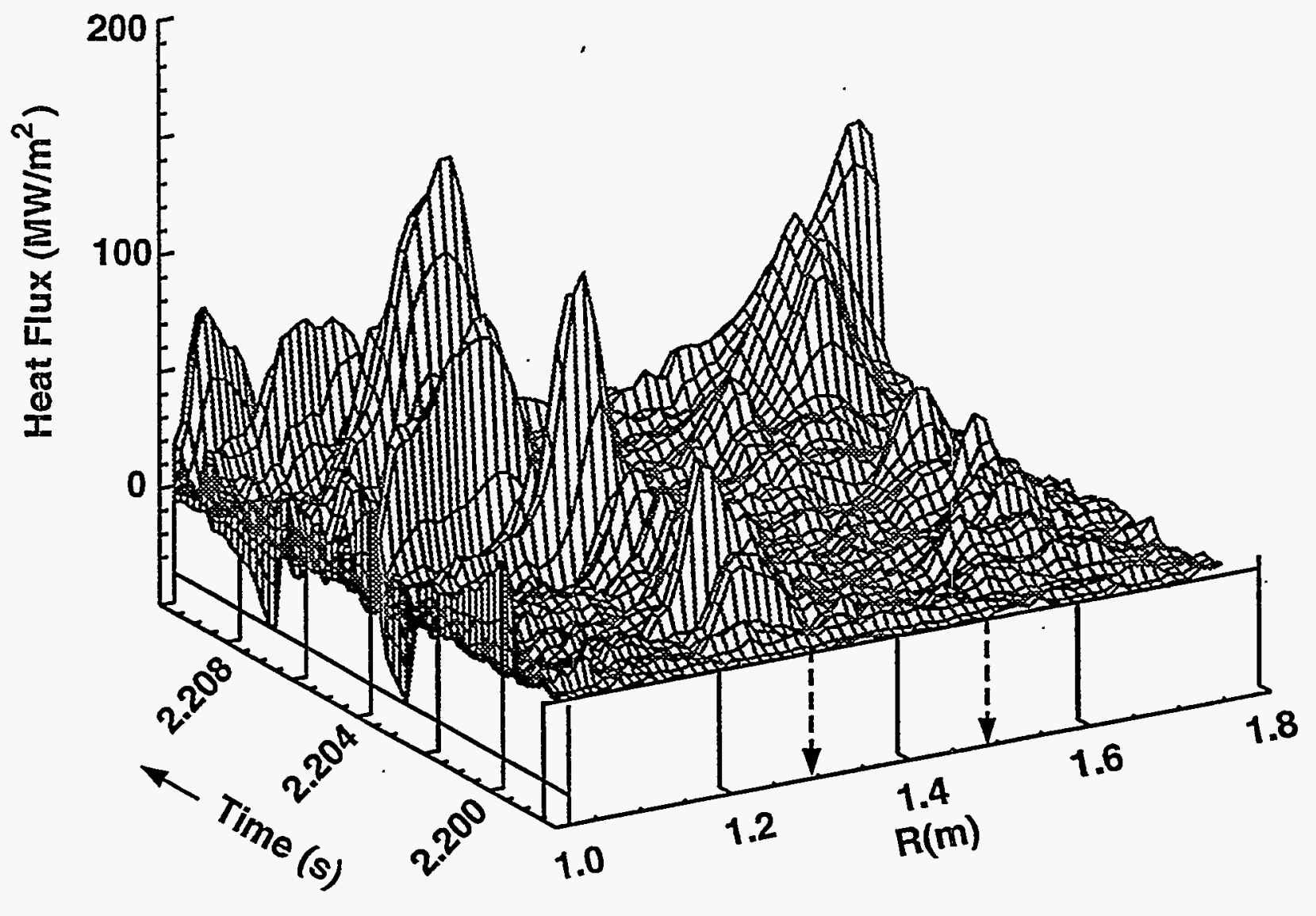

Fig. 2 

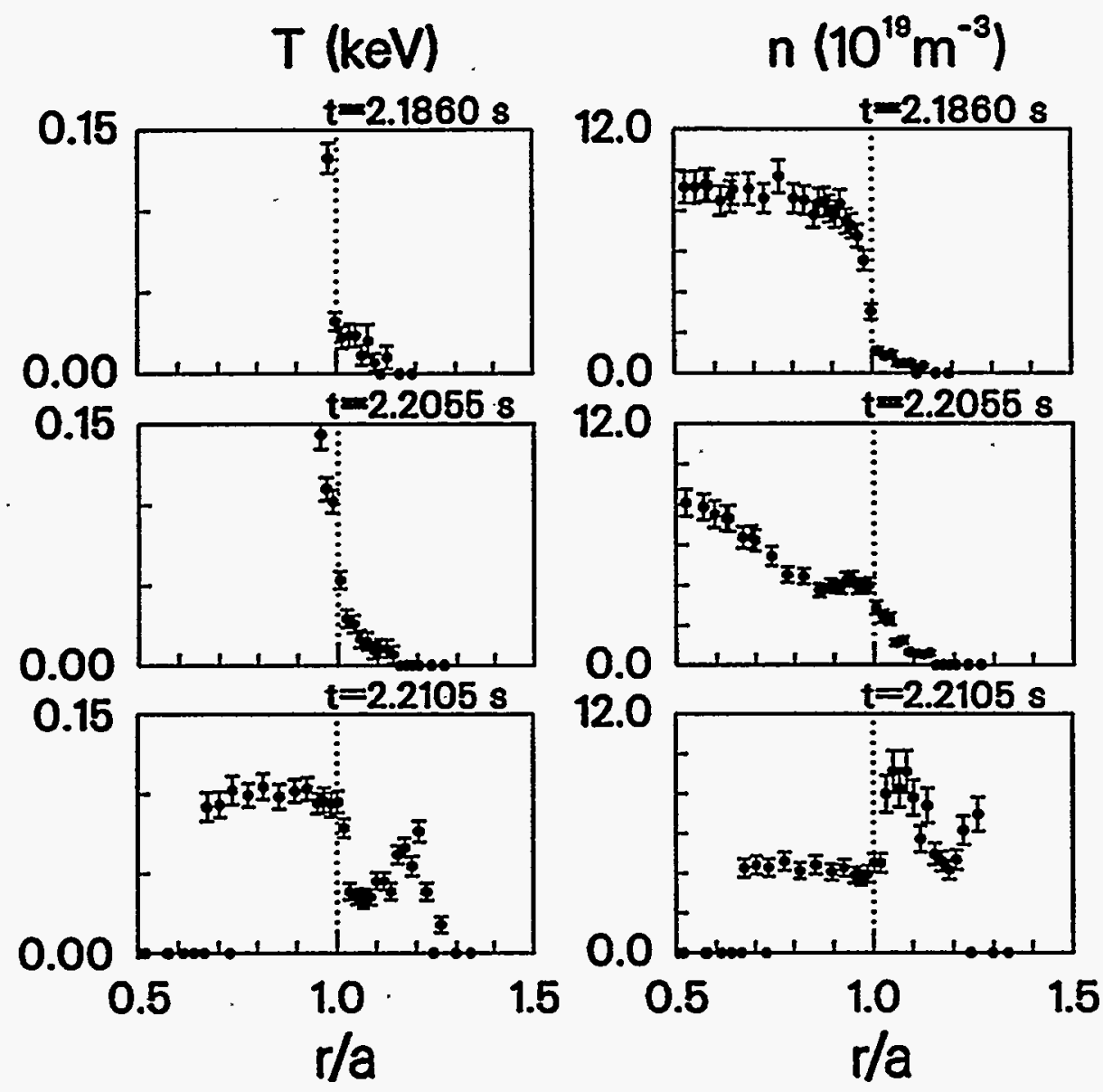

Fig. 3 


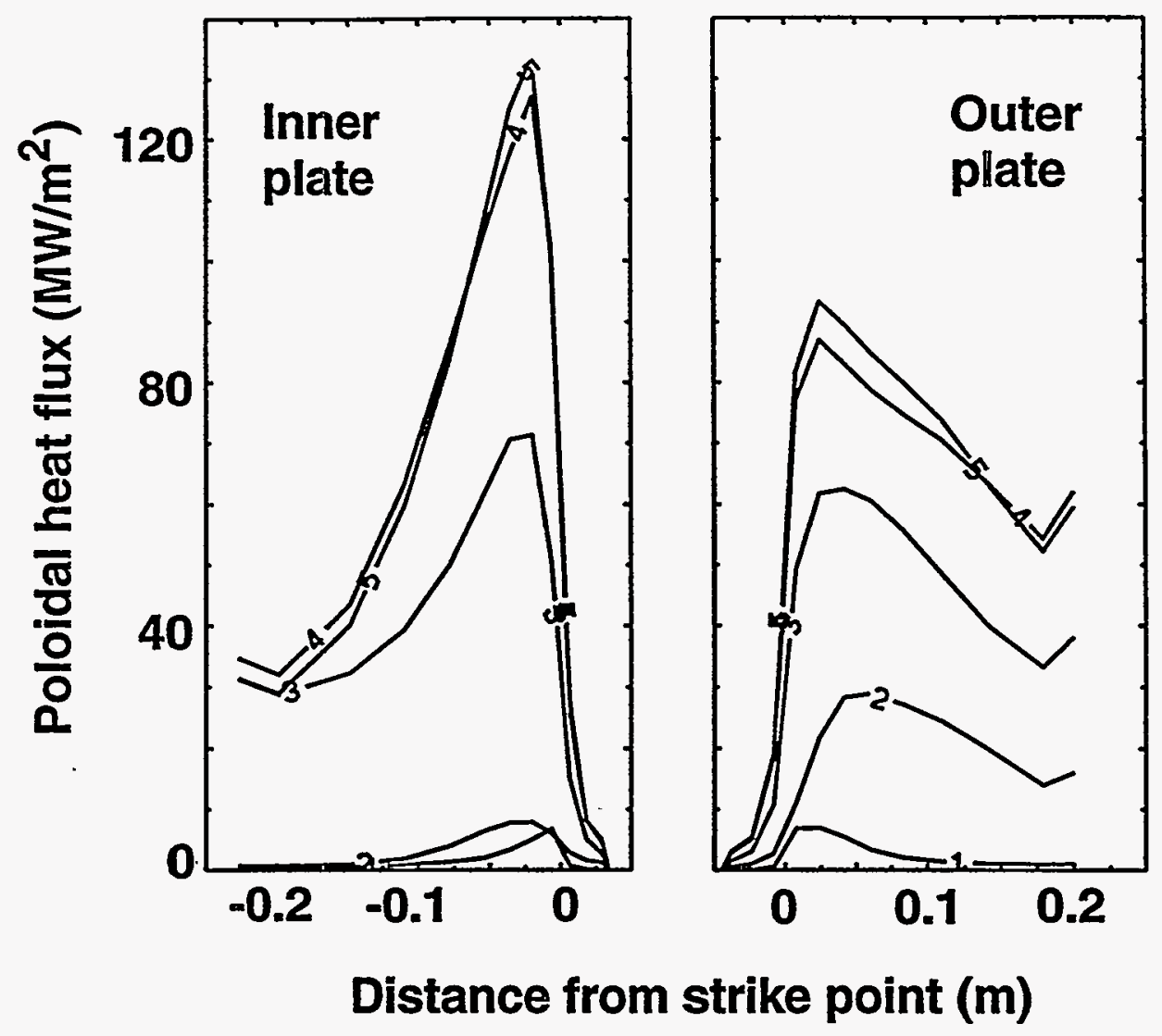

Fig. 4 


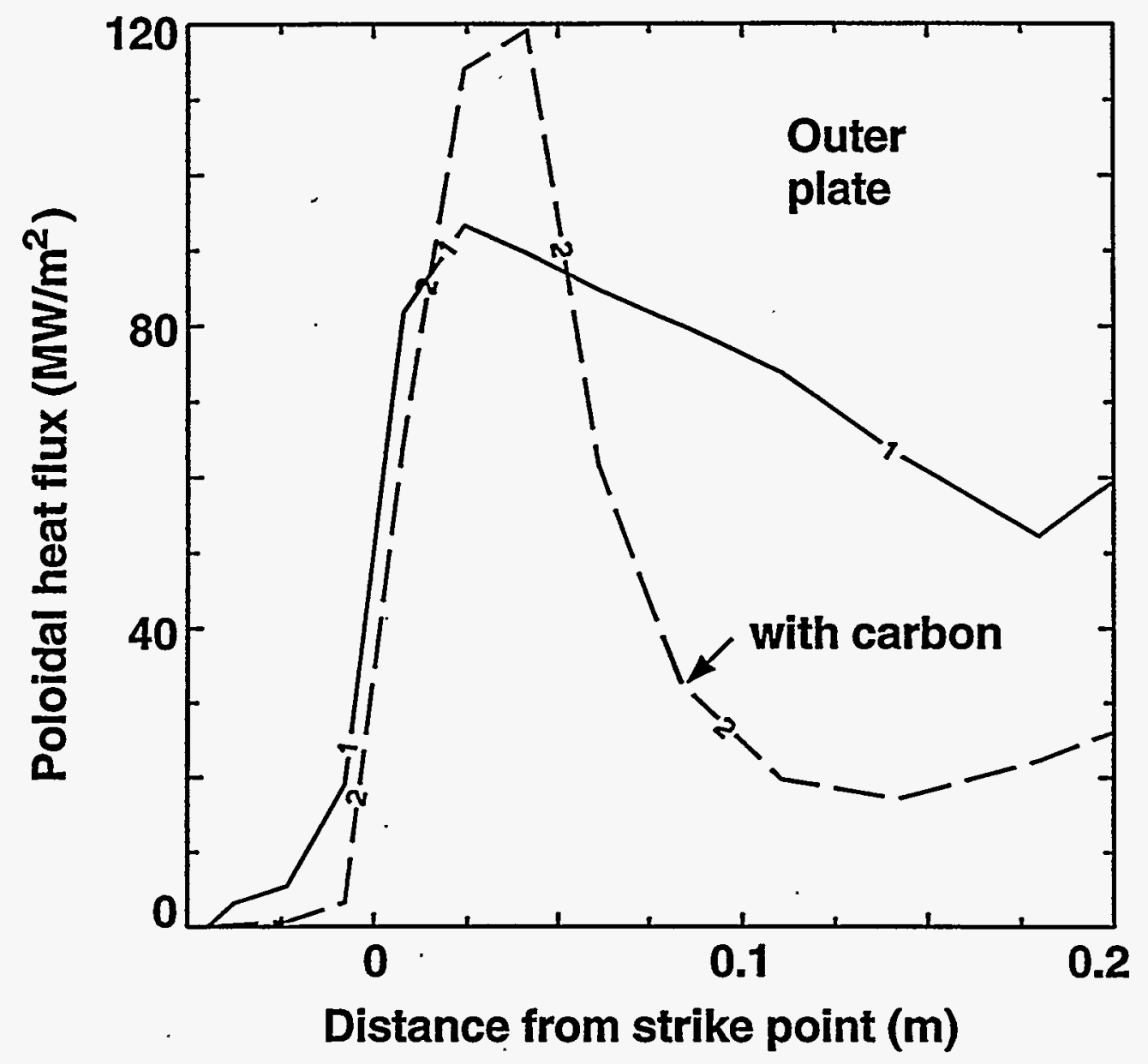

Fig. 5 


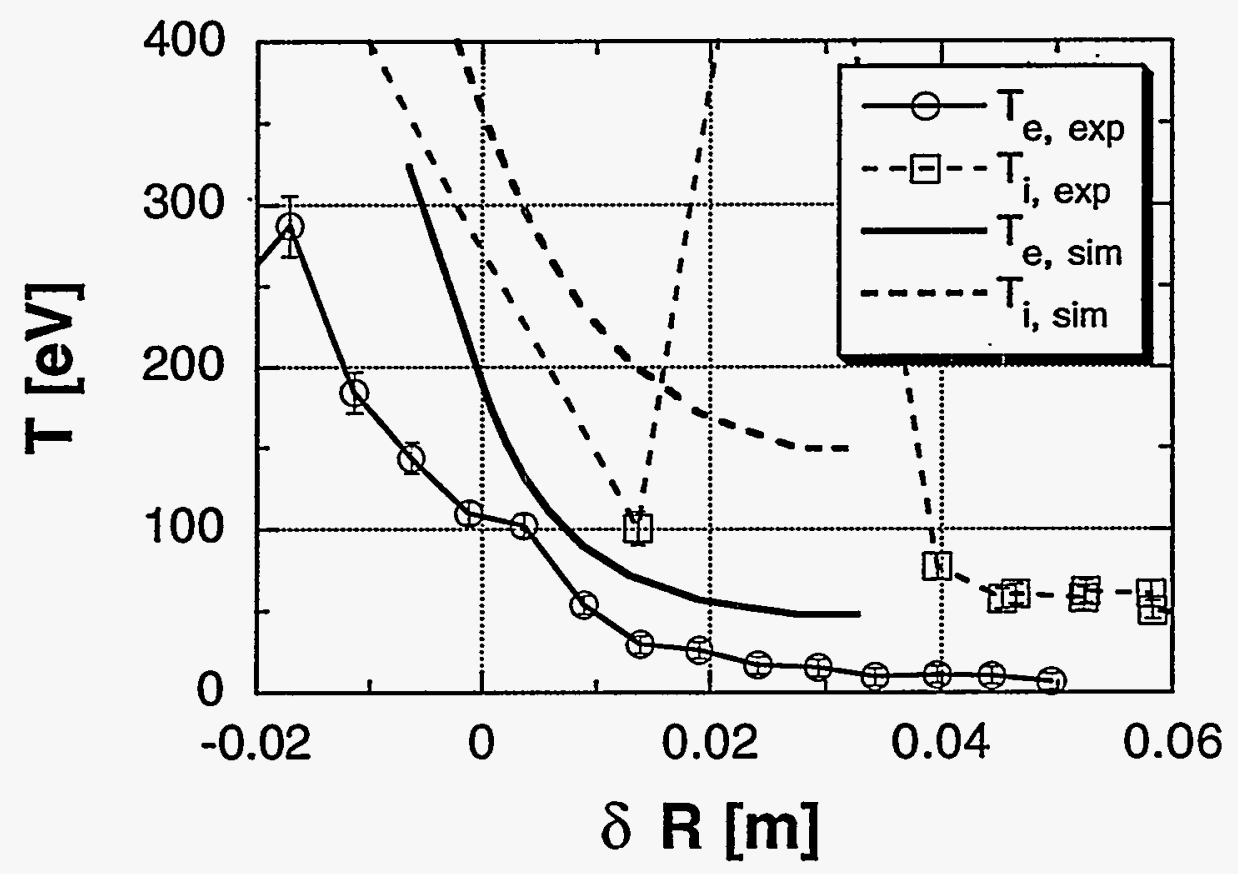

Fig.6 